This is the accepted version of the following article: Raiteri, P. and Bussi, G. and Cucinotta, C. and Credi, A. and Stoddart, J. and Parrinello, M. 2008. Unravelling the Shuttling Mechanism in a Photoswitchable Multicomponent Bistable Rotaxane. Angewandte Chemie-International Edition. 47 (19): pp. 3536-3539, which has been published in final form at: http://doi.org/10.1002/anie.200705207 


\section{Unravelling the Shuttling Mechanism in a Photoswitchable Multicomponent Bistable Rotaxane**1}

Paolo Raiteri, ${ }^{*}$ Giovanni Bussi, Clotilde S. Cucinotta, Alberto Credi,* J. Fraser Stoddart, Michele Parrinello. ${ }^{2}$

Much effort is being devoted currently to the bottom-up construction and operation of multicomponent molecular systems capable of performing specific, directional mechanical movements under the action of a defined energy input namely, molecular machines. $\left.{ }^{[i, i]}\right]$ This research not only constitutes a fascinating challenge in the field of nanoscience, but could also find applications in many fields of technology and medicine. However, despite the large number of molecular machines that have been constructed,, i,ii,iii,iv] a rather limited number of studies, aimed at elucidating the details of the working mechanism and the factors that influence the molecular motions in this kind of systems, are available.[v] In this Communication we present the results of a computational investigation on the operation mechanism of a photoswitchable bistable rotaxane that functions as an autonomous molecular shuttle powered by visible light. [vi] The role of the counteranions, and the shuttling free energy and rates are successfully unravelled by a multi-scale approach and our findings have been validated by experimental observations. Intriguing properties that have not (yet) been observed are predicted, thereby suggesting directions for future experimentation.

The bistable rotaxane, $\mathbf{1}^{6+}$ (Scheme 1 ), was specifically designed[vii] to achieve photoinduced ring shuttling in solution. This compound has a modular structure; its ring component $\mathrm{R}$ is a $\pi$-electron donating bis- $p$ phenylene[34]crown-10 macrocycle, whereas its dumbbell component is made

\footnotetext{
${ }^{1}$ We thank prof. V. Balzani and M. Venturi for discussions. Financial support from the EU (Biomach project), Ministero dell'Università e della Ricerca (PRIN 2006034123_003) and Università di Bologna is gratefully acknowledged. The CSCS is gratefully acknowledged for the computer time. The graphical material has been produced by M. Valle at the CSCS Visualization Group. Supporting information for this article is available on the WWW under http://www.angewandte.org or from the author.

${ }^{2}$ Dr. P. Raiteri, Dr. G. Bussi, Dr. C. S. Cucinotta, Prof. M. Parrinello

Computational Science, Dept. of Chemistry and Applied Biosciences

ETH Zurich

c/o USI Campus, Via G. Buffi 13, 6900 Lugano, Switzerland

Fax: +41(0)58666 4817

praiteri@ethz.ch

Prof. A. Credi

Dipartimento di Chimica "G. Ciamician"

Università di Bologna

via Selmi 2, 40126 Bologna, Italy

Fax: (+)39-051-2099456

E-mail: alberto.credi@unibo.it

Prof. J.F. Stoddart

California NanoSystems Institute

and Department of Chemistry and Biochemistry

University of California, Los Angeles

405 Hilgard Avenue, Los Angeles, CA 90095-1569, USA

Fax: (+)1-310-206-5621

E-mail: stoddart@chem.ucla.edu
} 
of several covalently linked units. They are a $\mathrm{Ru}^{\mathrm{II}}$ polypyridine complex $\left(\mathrm{P}^{2+}\right)$ as the photosensitizer and stopper, a $p$-terphenyl-type rigid spacer (S), a 4,4'bipyridinium $\left(\mathrm{A}_{1}{ }^{2+}\right)$ and a 3,3'-dimethyl-4,4'-bipyridinium $\left(\mathrm{A}_{2}{ }^{2+}\right) \pi$-electron accepting stations, and a tetraarylmethane group as the terminal stopper (T). The Ru-based unit plays the dual role of a light-fueled power station and a stopper, whereas the mechanical switch consists of the two electron accepting stations and the electron-donating ring. Six hexafluorophosphate ions $\left(\mathrm{PF}_{6}^{-}\right)$are present as the counteranions of the positively charged rotaxane. The stable translational isomer of $\mathbf{1}^{6+}$ in the ground state is the one in which the $R$ component encircles the $\mathrm{A}_{1}{ }^{2+}$ unit, in keeping with the fact that this station is a better electron acceptor than the other one.

The strategy devised in order to obtain the photoinduced shuttling movement of $\mathrm{R}$ between the two stations $\mathrm{A}_{1}{ }^{2+}$ and $\mathrm{A}_{2}{ }^{2+}$ is based on a 'four stroke' synchronized sequence of electronic and nuclear processes, as illustrated in Scheme 1. [vi] Light excitation of the photoactive unit $\mathrm{P}^{2+}$ (process 1 ) is followed by the transfer of an electron from this unit to $\mathrm{A}_{1}{ }^{2+}$ (process 2) which competes with the intrinsic decay of the $\mathrm{P}^{2+}$ excited state (process 3 ). After the reduction of $\mathrm{A}_{1}{ }^{2+}$, with the consequent 'deactivation' of this station, the ring moves (process 4) by $1.3 \mathrm{~nm}$ towards $\mathrm{A}_{2}{ }^{2+}$, a step that is in competition with the back electrontransfer from $\mathrm{A}_{1}{ }^{+}$(still encircled by $\mathrm{R}$ ) to the oxidized unit $\mathrm{P}^{3+}$ (process 5). Eventually, a back electron-transfer from the 'free' reduced station $\mathrm{A}_{1}{ }^{+}$to the oxidized unit $\mathrm{P}^{3+}$ (process 6) restores the electron acceptor power to this radical cationic station. As a consequence of the electronic reset, thermally activated back movement of the ring from $\mathrm{A}_{2}{ }^{2+}$ to $\mathrm{A}_{1}{ }^{2+}$ takes place (process 7). Steady-state and time-resolved spectroscopic experiments, together with electrochemical measurements in acetonitrile solution, showed[vi] that the absorption of a visible photon by $\mathbf{1}^{6+}$ can cause the occurrence of a forward and backward ring movement - that is, a full mechanical cycle according to Scheme 1.

From the computational point of view, direct simulations of the photoexcitation and of the electron transfer in such a large molecule is not feasible and, additionally, it would provide limited insight into the mechanism of the molecular motion. It is therefore more profitable to start analyzing the system just after the electron transfer has occurred, and focus on the ring displacement. The time scale of the shuttling movement and the complexity of the system require a multi-scale approach. The general picture can indeed be obtained only by enlarging our focus progressively from a quantum mechanical (QM) treatment of the charged stations to a molecular mechanical (MM) description of $\mathbf{1}^{6+}$ in acetonitrile, and eventually to a coarse grained (CG) description of several rotaxane molecules in solution.

Simple steric considerations reveal that the counteranions, if bonded to the axle of the dumbbell, may act as a hindrance to the shuttling movement and hence play an important role in determining the final efficiency of the molecular shuttle. QM calculations performed with GAUSSIAN03[viii] using the B3LYP/6$31 \mathrm{~g}^{*}$ approach and the Polarizable Continuum Model ${ }^{[i x]}$ to include the effects of the solvent indicate a binding energy of 11.7 and $10.3 \mathrm{kcal} \mathrm{mol}^{-1}$ between a $\mathrm{PF}_{6}{ }^{-}$ ion and the charged fragments of the dumbbell in the oxidized (ground) state (Figure 1) and in the reduced state afforded by light excitation, respectively. A coarse grained (CG) model composed of charged rods (mimicking $\mathbf{1}^{6+}$ ) and spheres $\left(\mathrm{PF}_{6}^{-}\right)$at the experimental density $(0.1 \mathrm{mM})$ can then be used to unravel 
the interplay between entropic and electrostatic effects that favor the dispersion of the $\mathrm{PF}_{6}{ }^{-}$ions against the formation of a bonded state (see SI). Long time CG simulations with DL_POLY2.16 ${ }^{[\mathrm{x}]}$ in which the interaction between the rods and the spheres is strengthened progressively reveal that, when the binding energy between the rods and the spheres overcomes $13 \mathrm{kcal} \mathrm{mol}^{-1}$, there is a dynamical exchange among the $\mathrm{PF}_{6}{ }^{-}$ions, although at least one anion is always bonded to the dumbbell. The high binding energy (compatible with the experimental estimate for the shuttling barrier of $\left.12 \mathrm{kcal} \mathrm{mol}^{-1}\right)^{[\mathrm{vi}]}$ and the CG results indicate that the detachment of the counteranion is a slow process and hence it might limit the efficiency of the nanomachine, in agreement with experimental observations on related systems. ${ }^{[\mathrm{vc}, \mathrm{xi}]}$

Further analysis of the QM calculations reveals that the strong interaction between the bistable rotaxane and counteranions arises mainly from polarization effects and a sizeable charge transfer $(0.17 e)$ from the $\mathrm{PF}_{6}{ }^{-}$ion to the axle. As these effects cannot be reproduced by a MM force field, in the following simulations we constrain the $\mathrm{PF}_{6}{ }^{-}$ions away from the dumbbell and focus on the sole contribution of the ring displacement to the overall shuttling rate. The time scale necessary to simulate the shuttling process directly is hardly achievable even by MM simulations. However, a quantitative comparison with experiments can be obtained by calculating the MM free energy profile and then the shuttling rate by solving the Fokker-Planck equation. [xii] The ring displacement can be easily described by considering the projection of the centre of mass of the ring $\mathrm{R}$ on the axle of the dumbbell. The free energy profile along this reaction coordinate $(L)$ (Figure 2) is then readily calculated using the Weighted Histogram Analysis Method ${ }^{[x i i i]}$ (see SI for further details). The relative stabilities of the two co-conformers in both oxidation states (Figure 2) agrees perfectly with the experimental estimates ${ }^{[\mathrm{vi}]}$ whilst the absence of any relevant barrier for the ring displacement from $\mathrm{A}_{1}{ }^{+}$(Figure 2, bottom) is evident. We then use these free energies as inputs for a statistical description of the dynamics of $L$ with the Fokker-Planck equation (see SI). The calculated time constant for ring shuttling from $\mathrm{A}_{1}{ }^{+}$to $\mathrm{A}_{2}{ }^{2+}$ is $\sim 20 \mathrm{~ns}$ at $300 \mathrm{~K}$, which is more than two thousand times faster than the measured one ( $\left.47 \mathrm{~ms}^{[\mathrm{vi}]}\right)$. Given the complexity and flexibility of the system, this is a highly unexpected result: it suggests that the pure ring displacement is not curbing the shuttling process, and hence is not crucial for determining the efficiency of the molecular machine. ${ }^{[x i v]}$

In summary, the free energy calculations confirm the experimental findings about the relative stability of the different co-conformations in the two oxidation states of the bistable rotaxane. However, the observed shuttling kinetics cannot be reproduced computationally if the role of the counteranions is not explicitly taken into account. We have indeed shown that the anions are strongly bonded to the axle of the dumbbell component, and that their detachment from the rotaxane framework may be the rate-limiting step in the translational isomerization process. We have also demonstrated that the shuttling movement is almost barrierless in the absence of the counteranions. Therefore, if the interaction between the positively charged rotaxane and its counteranions could be weakened, e.g., by changing the nature of the solvent or the counteranion, this molecular shuttle might work as a fast-switching, powerstroke nanomachine. ${ }^{[\mathrm{xv}, \mathrm{xvi}]}$ Experiments in this direction are not straightforward because of solubility issues and difficulties related to counteranion exchange for 
$\mathbf{1}^{6+}$. Nonetheless, experiments are already underway in our laboratories to test this hypothesis.

Keywords: Molecular Machine, Nanomotor, Multiscale Molecular Dynamics, Free Energy, Fokker-Planck Equation. 


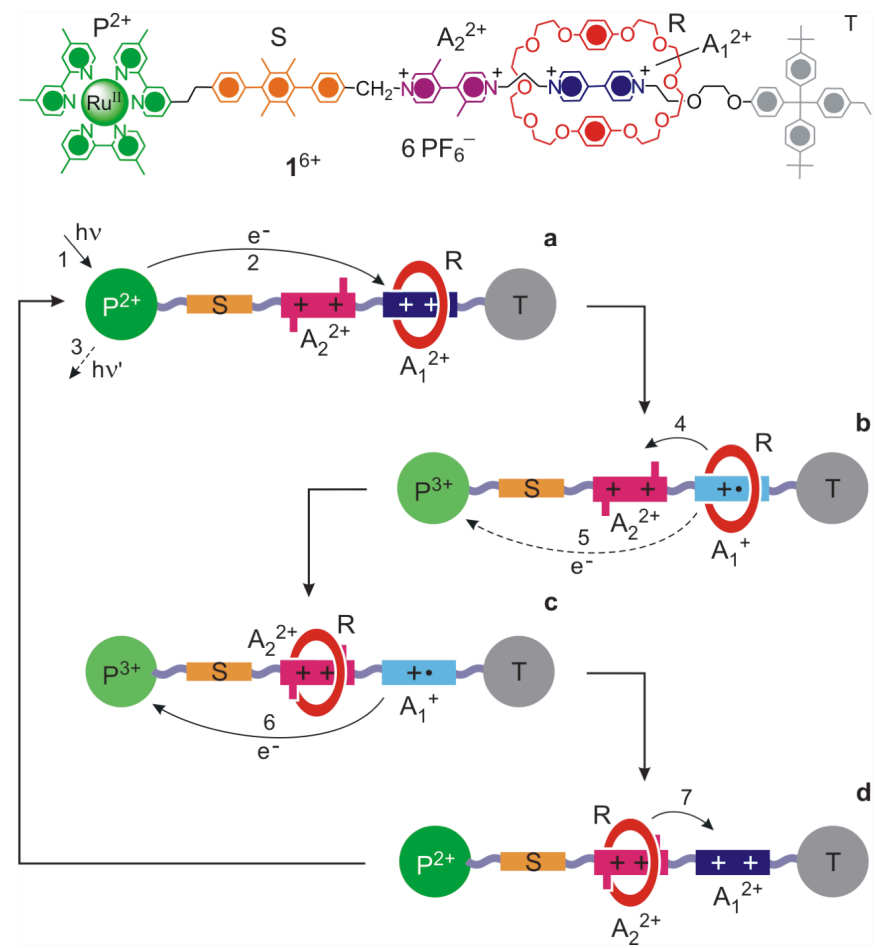

Scheme 1. Structure formula of rotaxane $\mathbf{1}^{6+}$ and a graphical representation of its working mechanism as an autonomous 'four stroke' molecular shuttle powered by visible light: $a$, destabilization of the stable translational isomer; $b$, ring displacement; c, electronic reset; $d$, nuclear reset. 


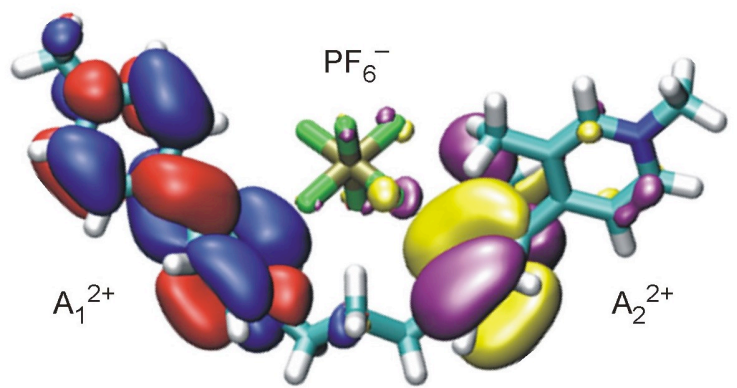

Figure 1. Graphical representation of the HOMO (yellow/purple) and LUMO (red/blue) molecular orbitals for the complex formed between a $\mathrm{PF}_{6}{ }^{-}$ion and the $\mathrm{A}_{1}{ }^{2+}$ and $\mathrm{A}_{2}{ }^{2+}$ stations. The LUMO is located on $\mathrm{A}_{1}{ }^{2+}$, in agreement with the experimental electron affinity of the two stations. The strong interaction between $\mathrm{A}_{2}{ }^{2+}$ and $\mathrm{PF}_{6}{ }^{-}$is evidenced by the localization of the HOMO on both fragments. In order to reduce the computational workload, only the $\mathrm{PF}_{6}{ }^{-}$ion and the charged stations have been considered. Preliminary calculations with the macrocycle suggest a further increase of the binding energy. 


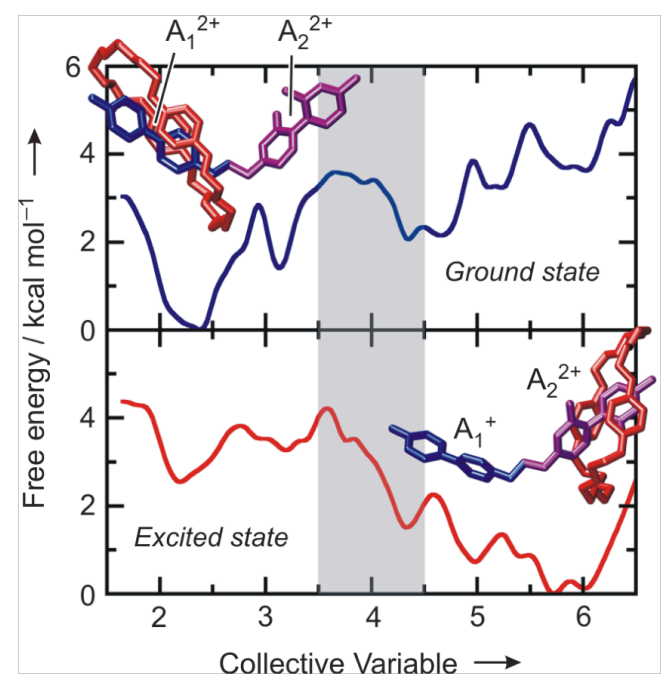

Figure 2. Free energy profile computed for the oxidized (ground) state (top; see Scheme 1a) and the reduced state afforded by photoexcitation (bottom; see Scheme 1c) of $\mathbf{1}^{6+}$ as a function of a collective variable (CV) which describes the position of the ring along the axle of the dumbbell. The shaded area indicates the $C V$ region in which the ring $R$ encircles the hinge between $A_{1}$ (left) and $A_{2}$ (right). The insets show the typical atomic arrangement corresponding to the two coconformers. The rotaxane is placed in a box of $\sim 560 \mathrm{~nm}^{3}$ filled with acetonitrile molecules $(\sim 36,000$ atoms). The simulations are performed with the all atom AMBER99[xvii] force field and the NAMD $^{[x v i i]}$ code at $300 \mathrm{~K}$ and 1 bar. The overall simulation time for the free energy calculation is more than 200 ns and the statistical analysis of the data confirms that all the orthogonal degrees of freedom are correctly sampled and that the accuracy on the profile is of the order of $k_{\mathrm{B}} T$. 
[i] V. Balzani, A. Credi, M. Venturi, Molecular Devices and Machines - Concepts and Perspectives for the Nanoworld, Wiley-VCH, Weinheim, 2008.

[ii] E.R. Kay, F. Zerbetto, D.A. Leigh, Angew. Chem. Int. Ed. 2007, 46, 72-191.

[iii] a) V. Balzani, A. Credi, F.M. Raymo, J.F. Stoddart, Angew. Chem. Int. Ed. 2000, 39, 3348-3391; b) K. Kinbara, T. Aida, Chem. Rev. 2005, 105, 1377-1400;

c) G.S. Kottas, L.I. Clarke, D. Horinek, J. Michl, Chem. Rev. 2005, 105, 1281-1376.

d) H. Tian, Q.C. Wang, Chem. Soc. Rev. 2006, 35, 361-374; e) W.R. Browne, B.L. Feringa, Nat. Nanotech. 2006, 1, 25-35; f) S. Saha, J.F. Stoddart, Chem. Soc. Rev. 2007, 36, 77-92; g) B. Champin, P. Mobian, J.-P. Sauvage, Chem. Soc. Rev. 2007, 36, 358-366.

[iv] a) Acc. Chem. Res. 2001, 34(6), special issue on Molecular Machines (Ed.: J.F. Stoddart); b) Struct. Bond. 2001, 99, volume on Molecular Machines and Motors (Ed.: J.-P. Sauvage); c) Top. Curr. Chem. 2005, 262, volume on Molecular Machines (Ed.: T. R. Kelly); d) Org. Biomol. Chem. 2007, 4(18), special issue on DNA Nanomachines (Ed.: I. Willner); e) Adv. Funct. Mater. 2007, 17(5), special issue on Molecular Machines and Switches (Eds.: A. Credi, H. Tian).

[v] a) D.A. Leigh, A. Troisi, F. Zerbetto, Chem. Eur. J. 2001, 7, 1450-1454; b) N. Koumura, E.M. Geertsema, M.B. van Gelder, A. Meetsma, B.L. Feringa, J. Am. Chem. Soc. 2002, 124, 5037-5051; c) S. Garaudée, S. Silvi, M. Venturi, A. Credi, A.H. Flood, J.F. Stoddart, ChemPhysChem 2005, 6, 2145-2152; d) S.S. Jang, et al., J. Am. Chem. Soc. 2005, 127, 1563-1575; e) M.K.J. ter Wiel, R.A. van Delden, A. Meetsma, B.L. Feringa, J. Am. Chem. Soc. 2005, 127, 14208-14222; f) M. Ceccarelli, F.

Mercuri, D. Passerone, M. Parrinello, J. Phys. Chem. B 2005, 109, 17094-17099; g) J.W. Choi, et al., Chem. Eur. J. 2006, 12, 261-279; h) Y.-H. Yang, W.A. Goddard, J. Phys. Chem. B 2006, 110, 7660-7665; i) J. Vicario, M. Walko, A. Meetsma, B.L. Feringa, J. Am. Chem. Soc. 2006, 128, 5127-5135.

[vi] V. Balzani, M. Clemente-León, A. Credi, B. Ferrer, M. Venturi, A. H. Flood, J. F. Stoddart, Proc. Natl. Acad. Sci. USA 2006, 103, 1178-1183.

[vii] P.R. Ashton, et al., Chem. Eur. J. 2000, 6, 3558-3574.

[viii] Gaussian 03, Revision D.01, M. J. Frisch, et al., Gaussian, Inc., Wallingford CT, 2004.

[ix] M. Cozzi, G. Scalmani, N. Rega, V. Barone, J. Chem. Phys., 2002, 117, 43-54.

[x] W. Smith (Guest Editor), Molecular Simulation, 2006, 32, 933-1121.

[xi] A. Credi, S. Dumas, S. Silvi, M. Venturi, A. Arduini, A. Pochini, A. Secchi, J. Org. Chem. 2004, 69, 5881-5887.

[xii] C. W. Gardiner, Handbook of Stochastic Methods, Springer-Verlag, 2004.

[xiii] S. Kumar, J.M. Rosenberg, D. Bouzida, R.H. Swendsen, P.A. Kollman, J. Comp. Chem. 1992, 13, 1011-1021.

[xiv] The efficiency of ring displacement from $\mathrm{A}_{1}{ }^{+}$to $\mathrm{A}_{2}{ }^{2+}$, which in turn determines the quantum yield of the shuttling process, depends on the competition between processes 4 and 5 in Scheme 1b. In the present case, such an efficiency was determined to be only $12 \%$ at 303 K. For a detailed discussion, see ref. [vi].

[xv] H. Wang, G. Oster, Appl. Phys. A 2002, 75, 315-323.

[xvi] For a discussion on the design of a light-fueled nanomotor with a powerstroke mechanism, see: J. Vacek, J. Michl, Adv. Funct. Mater. 2007, 17, 730739. 
[xvii] J. Wang, P. Cieplak, P. A. Kollman, J. Comp. Chem. 2000, 21, 1049-1074.

[xviii] J.C. Phillips, R. Braun, W. Wang, J. Gumbart, E. Tajkhorshid, E. Villa, C.

Chipot, R.D. Skeel, L. Kale, K. Schulten, J. Comp. Chem. 2005, 26, 1781-1802. 